\title{
Combustion Blowoff Effects on the Central Recirculation Zone using various Syngas mixtures in a Tangential Swirl Burner
}

\author{
Baej H, Valera-Medina A, Syred N*, Marsh R, Bowen P \\ College of Physical Sciences and Engineering, Cardiff University, CF24 3AA \\ * Corresponding author. Email: syred@cf.ac.uk ;Tel. +44 (0)2920 875948
}

\begin{abstract}
Lean premixed combustion is one of the most successful technologies for flame control in low NOx systems. The characteristics of these flows its good mixing performance, stability and the low emissions. The potential of using new alternative fuels presents a problem in terms due to heating value changes, flame parameters and reactivity. Bio-renewable processes and industrial systems requiring waste gases are just a few examples. The biggest challenge to fuel-flexibility is the large differences between natural gas and the proposed alternative fuels which causes variations in the stability profiles of the combustion process.

In this paper, combustion of $\mathrm{CH}_{4} / \mathrm{H}_{2} / \mathrm{CO}$ mixtures was experimentally and numerically studied to understand the impacts of these fuels on the blowoff process. Atmospheric pressure and ambient temperature were used at moderate swirl number. Various nozzles were used to determine the impact of the blends on the Central Recirculation Zones. Methane content in the fuel was decreased from $50 \%$ to $0 \%$ (by volume) with the remaining amount split equally between carbon monoxide and hydrogen. The Central Recirculation Zone and its turbulence were numerically characterised using the $\mathrm{k}-\omega$ turbulence model providing details of the structure close to blowoff. The results show how the strength and size of the recirculation zone are highly influenced by the blend, carbon/hydrogen ratio, nozzle geometry and Re numbers.
\end{abstract}

Keywords: Central Recirculation Zone, Damkölher number, CFD, Precessing Vortex Core.

\section{Nomenclature}

\begin{tabular}{|l|l|l|l|}
\hline S & Swirl number [-] & G & Stretch factor [-] \\
\hline CRZ & Central Recirculation Zone & erfc & Complementary error function \\
\hline $\mathrm{Da}$ & Damköhler number [-] & $\sigma$ & standard deviation of the distribution of $\epsilon$ \\
\hline$\tau_{\mathrm{t}}$ & Turbulent time scale $[\mathrm{s}]$ & $\mathrm{g}_{\mathrm{cr}}$ & Critical rate of strain[-] \\
\hline$\tau_{\mathrm{c}}$ & Chemical time scale $[\mathrm{s}]$ & $\mu_{\text {str }}$ & stretch factor coefficient for dissipation pulsation \\
\hline$\alpha$ & Thermal diffusively M2/s & $\mathrm{L}$ & Turbulent integral length \\
\hline $\mathrm{U}_{\mathrm{i}}$ & Laminar flame speed [m/s] & $\boldsymbol{\eta}$ & Kolmogorov micro-scale \\
\hline$\phi$ & Equivalences ratio & LBO & Lean blowoff \\
\hline PVC & Precessing vortex core & HP & High Power $=11.477 \mathrm{KW}$ \\
\hline LP & Low Power $=3.499 \mathrm{KW}$ & MP & Medium Power $=7.488 \mathrm{KW}$ \\
\hline
\end{tabular}

\section{Introduction}

Flame stabilization in premixed systems is an important topic of study for gas turbine combustors and industrial furnaces. Lean premixing combustion technologies (LPM) focus on operation at very low equivalence ratios in order to reduce thermal NOx production. However, new alternative fuels for gas turbines pose new challenges to the technology. The biggest challenge is the large differences between natural gas and the proposed replacement fuels. Moreover, the systems must meet current emission regulations, which often mean running at ultra-lean conditions near blowoff limits. However, blowoff is still a phenomenon difficult to predict when alternative blends are used. To 
describe the lean blowoff behaviour any burner running on alternative fuel compositions, correlations have to be determined and simplified models developed [1].

Most current gas turbines use swirl stabilized combustion, as it stabilises the burning process by anchoring the flame [2]. The crucial feature of swirl burners is a central recirculation zone (CRZ) which extends blowoff limits by recycling heat and active chemical species [3-4]. Thus, the CRZ is one of the mechanisms for flame stabilization that creates a point where the local flame speed and flow velocity match [5].

A significant amount of literature exists on measuring, correlating and predicting blowoff limits for swirl stabilized combustors. Longwell et al [6] suggested that blowoff occurs when it is not possible to balance the rate of entrainment of reactants into the recirculation zone and the subsequent turbulent burning velocity of the mixture. Since entrainment rates scale as the size of the CRZ increases and velocity of the flow is decreased, then it follows that this criterion reduces to a Damköhler number (Da) blowoff criterion, using a chemical time that is derived from the well stirred reactor theory [6]. As an example, most practical swirl combustion reactions take place at the lower limit, i.e. where Da $<<1$, so the turbulence timescales are significantly shorter than the chemistry.

There is general agreement that the blowoff process is controlled by a competition between the fluid mechanical and chemical kinetic processes, which can subsequently be defined in terms of a Damköhler number. A different view is that the contact time between the combustible mixture and hot gases in the shear layer must exceed a certain chemical ignition time. This implies a direct link between the scales of the characteristic dimension of the recirculation zone length, leading to a similar Da criterion [7]. Current theories are based on a flamelet based description upon local extinction by excessive flame stretch [8]. Flame stretching starts blowoff with the initiation of holes in the flame that are healed by the same flame creating stretching in areas that otherwise would have been unaffected. The flame will extinguish when the stretch rate exceeds a critical value [7]. However, limited work has been done on the impacts of the Precessing Vortex Core (PVC) and its interaction with the CRZ through the blowoff process. Previous experiments [9] have shown that turbulence close to the burner mouth can threefold as a consequence of the co-existence of both structures.

Fuel composition also plays an important role in this phenomenon. Research by Peters [10] resulted in a theory of turbulent premixed flames that becomes generally accepted in the combustion research community. The structure of a turbulent premixed flame is to be seen as superimposing instantaneous contours of convoluted reaction zones. The appearance of the reaction zone depends heavily on the governing turbulent structures and the chemical properties of the flow. The experiments conducted by Lieuwen et al. [11] to investigate $\mathrm{H}_{2} / \mathrm{CH}_{4}$ flames show that small additions of $\mathrm{H}_{2}$ substantially enhance the mixture's resistance to blowoff. Fundamental studies show that the extinction strain rate of methane flames is doubled with the addition of $10 \% \mathrm{H}_{2}$. Experiments were also conducted using $\mathrm{N}_{2}, \mathrm{H}_{2} \mathrm{O}$ and $\mathrm{CO}_{2}$. It was concluded that the flame speeds of mixtures with $\mathrm{CO}_{2}$ dilution are lower than those of mixtures diluted with chemically inert species with the same specific heat as $\mathrm{CO}_{2} \cdot \mathrm{CO}_{2}$ dilution can lead to lower laminar flame speeds and lower flame temperatures due to radiative losses from the flame [11]. The group showed that as the turbulence intensity increases, the turbulent flame speed initially increases. However, turbulence intensity and laminar flame speed alone do not capture many important characteristics of the turbulent flame speed. One must also consider the effects of flame instabilities and flame stretch [12]. Strakey et al [13] studied the effects of hydrogen addition in a lean-premixed swirl-stabilized combustor. They observed that increasing the hydrogen concentration in the fuel reduced blowoff in equivalence ratios from 0.46 to 0.30 . Schefer et al [14] found that hydrogen addition resulted in a significant change in the flame structure [14]. Nevertheless, the impact of coherent structures in the blowoff process with different blends has been barely analysed, leaving room for further studies.

Therefore, in this paper the structure of premixed swirl stabilized flames is analysed with the help of various numerical studies and experimental data, correlating the blowoff phenomenon with various syngas compositions to determine the influence of large coherent structures on the blowoff process. 


\section{Setup}

\section{Experimental Approach}

A generic swirl burner constructed from stainless steel was used to examine the flame stability limits under atmospheric conditions (1bar, 293K) at Cardiff University's Gas Turbine Research Centre (GTRC). A photograph and schematic of the generic burner is presented in Figure 1. More details can be found in previous literature [15]. A geometrical Swirl number, $S_{g}$, of 1.05 was used. The recirculation zone was distorted using a $30^{\circ}, 45^{\circ}$ and $60^{\circ}$ nozzles, Figure 2, as observed by ValeraMedina et al [4]. Confined and unconfined conditions were tested. Confinement was imposed using a quartz cylinder with a diameter of $3 \mathrm{D}$, being $\mathrm{D}$ the external nozzle diameter of $0.028 \mathrm{~m}$.
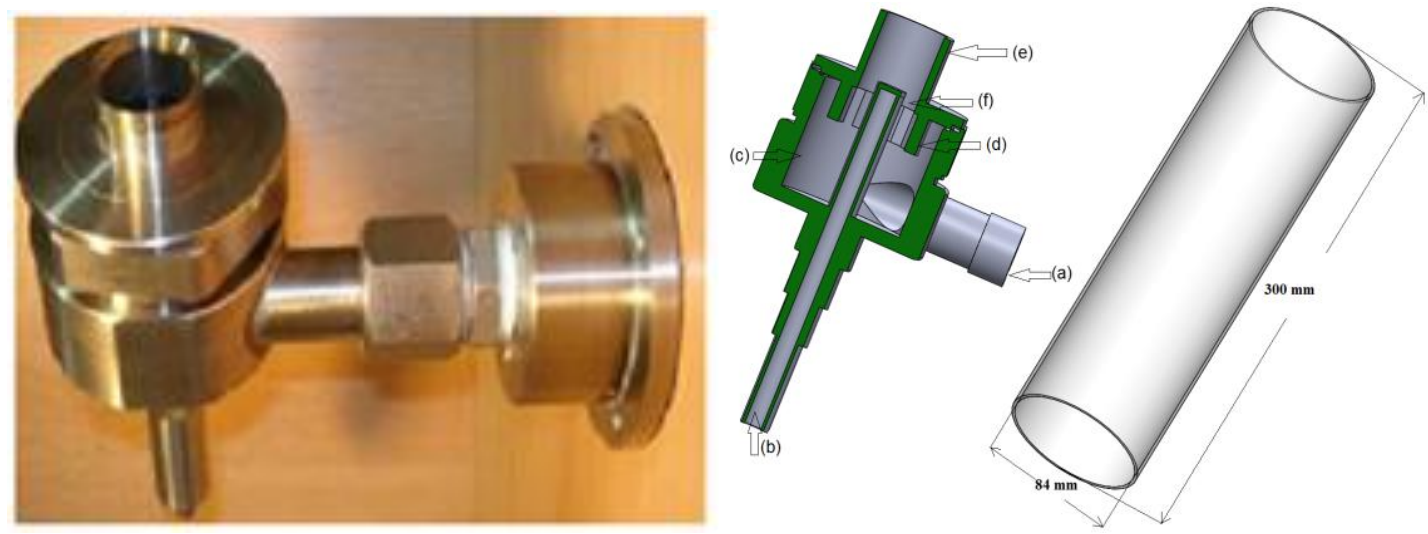

Figure 1: Unconfined swirl burner and schematic diagram and quartz tube respectively.
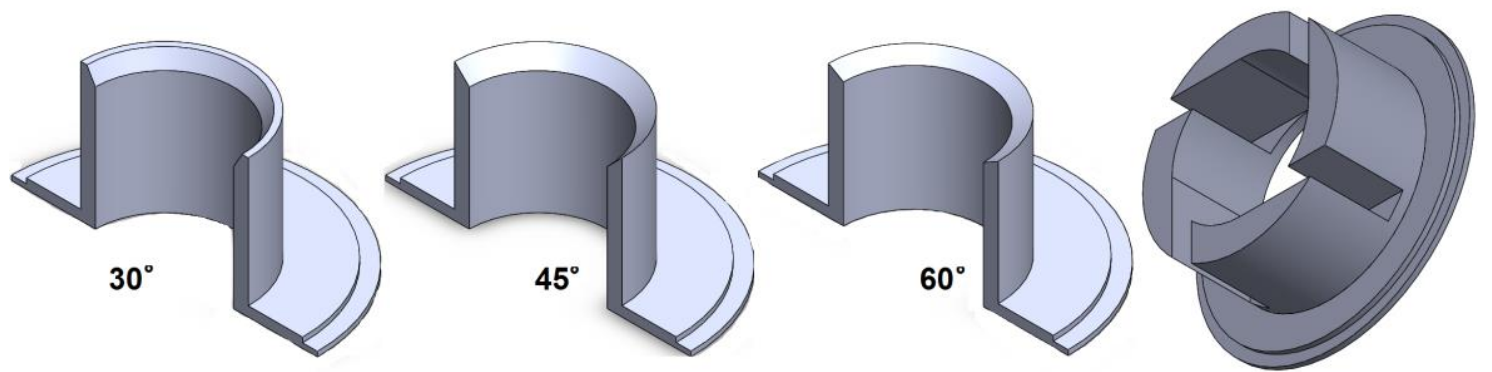

Figure 2: Angular nozzle and geometrical swirl respectively.

Experiments were conducted to define the blowoff limits of different configurations using different gases. The gases used were a mixture of $\mathrm{CH}_{4}, \mathrm{H}_{2}, \mathrm{CO}_{2}$ and $\mathrm{CO}$, Table 1 . The conditions analysed are presented in Table 2 .

Table 1: Syngas compositions

\begin{tabular}{|ll|}
\hline Gas number & Gas compositions \\
Syngas 1 & $10 \% \mathrm{CH}_{4}+45 \% \mathrm{H}_{2}+45 \% \mathrm{CO}$ \\
Syngas 2 & $20 \% \mathrm{CH}_{4}+40 \% \mathrm{H}_{2}+40 \% \mathrm{CO}$ \\
Syngas 3 & $30 \% \mathrm{CH}_{4}+35 \% \mathrm{H}_{2}+35 \% \mathrm{CO}$ \\
Syngas 4 & $50 \% \mathrm{CH}_{4}+25 \% \mathrm{H}_{2}+25 \% \mathrm{CO}$ \\
Gas 5 & $100 \% \mathrm{CH}_{4}$ \\
Gas 6 & $50 \% \mathrm{CH}_{4}+50 \% \mathrm{CO}_{2}$ \\
\hline
\end{tabular}

Table 2. Experimental and all CFD conditions at $7.49 \mathrm{~kW}$

\begin{tabular}{|cccccc|cccccc|}
\hline Gas & $\dot{M}$ fuel & $\dot{M}$ Air & $\alpha^{\circ}$ & Total & $\Phi$ & Gas & $\dot{M}$ fuel & $\dot{M}$ Air & $\alpha^{\circ}$ & Total & $\Phi$ \\
No & {$[g / s]$} & {$[g / s]$} & & {$[g / s]$} & & No & {$[g / s]$} & {$[g / s]$} & & {$[g / s]$} & \\
Syn1 & 0.101 & 1.41 & $30^{\circ}$ & 1.51 & 0.425 & Syn3 & 0.107 & 1.63 & $30^{\circ}$ & 1.73 & 0.563 \\
Syn1 & 0.101 & 1.40 & $45^{\circ}$ & 1.50 & 0.428 & Syn3 & 0.107 & 1.67 & $45^{\circ}$ & 1.78 & 0.548 \\
Syn1 & 0.101 & 1.38 & $60^{\circ}$ & 1.48 & 0.453 & Syn3 & 0.107 & 1.65 & $60^{\circ}$ & 1.75 & 0.557 \\
Syn2 & 0.104 & 1.55 & $30^{\circ}$ & 1.66 & 0.485 & Syn4 & 0.113 & 1.83 & $30^{\circ}$ & 1.95 & 0.689 \\
Syn2 & 0.104 & 1.55 & $45^{\circ}$ & 1.65 & 0.486 & Syn4 & 0.113 & 1.79 & $45^{\circ}$ & 1.90 & 0.707 \\
Syn2 & 0.104 & 1.48 & $60^{\circ}$ & 1.59 & 0.508 & Syn4 & 0.113 & 1.83 & $60^{\circ}$ & 1.94 & 0.692 \\
\hline
\end{tabular}




\section{Numerical Methodology}

During the simulation, various models were investigated and conclusions drawn as to which were the most effective. Based on the experimental results obtained at $1.485 \mathrm{~g} / \mathrm{s}$ to $1.946 \mathrm{~g} / \mathrm{s}$ the best option for the present work was the $\kappa-\omega$ SST model [16-18] with the following equations,

$$
\begin{aligned}
& \partial \rho / \partial t+\operatorname{div}(\rho U)=0 \\
& \left.\rho D_{u i} / D_{t}=-\partial p / \partial_{x i}\right)+\operatorname{div}\left(\mu \operatorname{grad} u_{i}\right)+S_{M i} \\
& \rho D E / D t=\operatorname{div}(\rho U)+\left[\left(\partial u \tau_{x x} / \partial_{x}+\partial\left(u \tau_{y x}\right) / \partial y+\partial\left(u \tau_{z x}\right) / \partial z+\partial\left(v \tau_{x y}\right) / \partial x+\partial\left(v \tau_{y y}\right) / \partial y+\right.\right. \\
& \left(\partial\left(v \tau_{z y}\right)\right) / \partial z+\partial\left(w \tau_{x z}\right) / \partial x+\partial\left(w \tau_{y z}\right) / \partial y+\partial\left(w \tau_{z z}\right) \\
& / \partial y]+\operatorname{div}(k \nabla \cdot T)+S_{E}
\end{aligned}
$$

The turbulent flame speed was computed using the following equation,

$$
U_{t}=A\left[u^{\prime}\right]^{\wedge 3 / 4}\left[U_{i}\right]^{\wedge 1 / 2} \alpha^{\wedge-1 / 4} l_{t^{\wedge}}^{\wedge / 4}=A u^{\prime}\left(\tau_{t} / \tau_{c}\right)^{\wedge}{ }^{1 / 4}
$$

Where the turbulent length scale $l_{t}$ is determined from $l_{t}=C_{D}\left(u^{\prime}\right)^{\wedge} / \varepsilon$

The default values of 0.52 for $A$ and 0.37 for $C_{D}$ are recommended by Zimont et al [20].

Therefore, the flame propagation can be modelled by solving transport equations of the weighted mean density reaction progress variable denoted by c based on the Zimont model [20]. In this model, the stretch factor $(\mathrm{G})$ represents the likelihood that the stretching will not quench the flame, i.e. if there is no stretching $(\mathrm{G}=1)$, the probability that the flame will be unquenched is $100 \%$. The stretch factor is obtained by integrating the log-normal distribution of turbulence dissipation rate, $\epsilon$ :

$$
\left.\mathrm{G}=1 / 2 \operatorname{erfc}\left\{-\sqrt{ } 1 / 2 \sigma\left[\operatorname{Ln}\left(\epsilon_{\mathrm{cr}} / \epsilon\right)\right]+\sigma / 2\right]\right\}
$$

The critical rate of strain $\left(\mathrm{g}_{\mathrm{cr}}\right)$ should be adjusted based on experimental data. For the numerical models an appropriate value can be determined as [19-20],

$$
\mathrm{g}_{\mathrm{cr}}=\mathrm{BU}_{\mathrm{i}}^{2} / \propto
$$

$\epsilon_{\mathrm{cr}}$, the turbulence dissipation rate at the critical rate of strain, is given by,

$\epsilon_{\mathrm{cr}}=15 v g^{2}$ cr

Simulations were performed using ANSYS Fluent 14.5 using these correlations. The pre-processor used to construct the mesh was ICEM 14.5.7. After independency mesh analyses, it was concluded that a medium size mesh of 796,878 elements would provide mesh independent results. Non-slip boundary conditions were defined using adiabatic conditions at 1 bar inlet pressure and inlet temperatures of $300 \mathrm{~K}$.

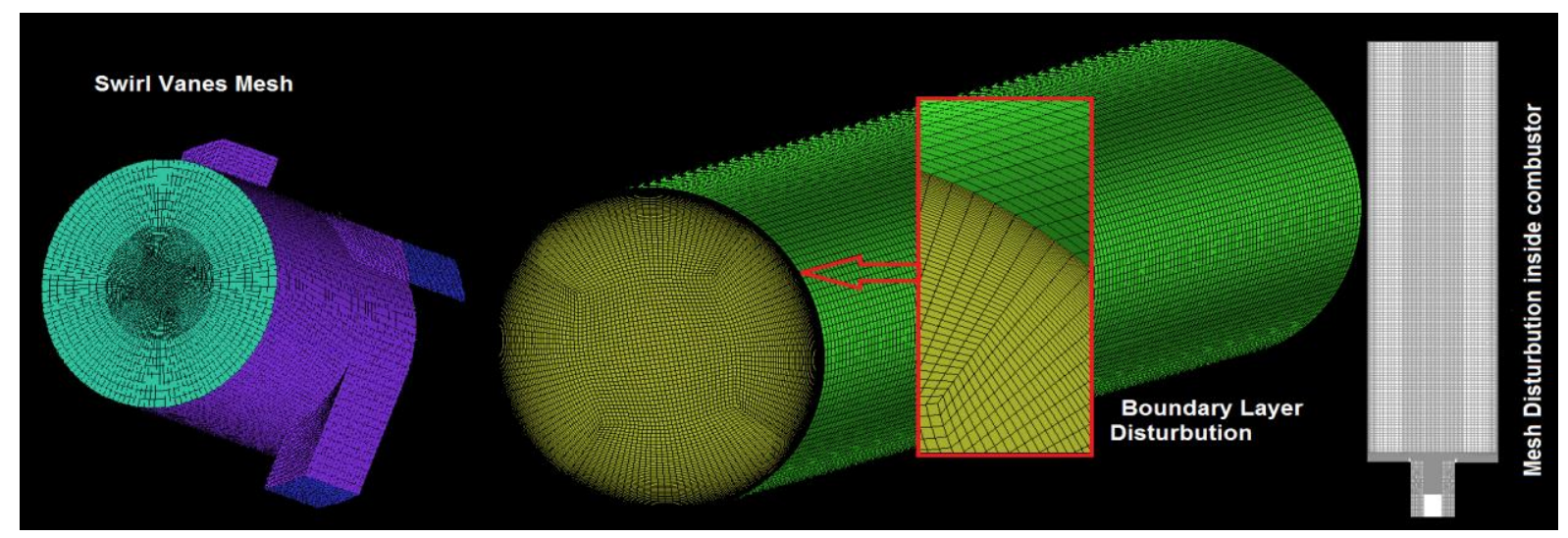

Figure 3: Mesh Distribution and boundary layer 


\section{Results \& Discussions}

Figure 4 shows the comparison between the 3 nozzles, Figure 2. As the mole fraction of hydrogen increases the equivalence ratio at which LBO occurs moves to leaner conditions, thus showing an improvement in blowoff limits, Figures 4. This has been proved elsewhere [13]. However, it can be seen that some trends follow linear progressions, especially during the experiments using the $45^{\circ}$ nozzle and syngas- 1 . As the angle is decreased/increased, the results become less linear, implying a breakdown in the controlling phenomena due to a more chaotic.

Similarly, the decrease in hydrogen produces less homogeneous results. This relates to the high reaction of hydrogen close to the dumping plane. The increase of $\mathrm{H}_{2}$ decreases the Da number as a consequence of faster chemical reactions. Thus, convective processes and turbulence produced at the burner nozzle do not appear to be controlling the onset of LBO. However, the reduction of hydrogen produces conditions with a more random behaviour towards the blowoff limit.

A comparison of blowoff limits, Figure 5, demonstrates the effects of the different fuels, plus data taken with pure methane and methane blended with carbon dioxide using a $45^{\circ}$ nozzle [15]. There is considerable improvement in LBO with the increase of hydrogen. On the other hand, syngas 4 with a $50 \%$ methane shows similar LBO values to pure methane. This demonstrates that although Da values are different between the two gases, blowoff occurs at the same equivalence ratios as a consequence of mechanisms that might be linked to coherent structures whose impact has greater effects on the blowoff limit at slower reaction time scales.

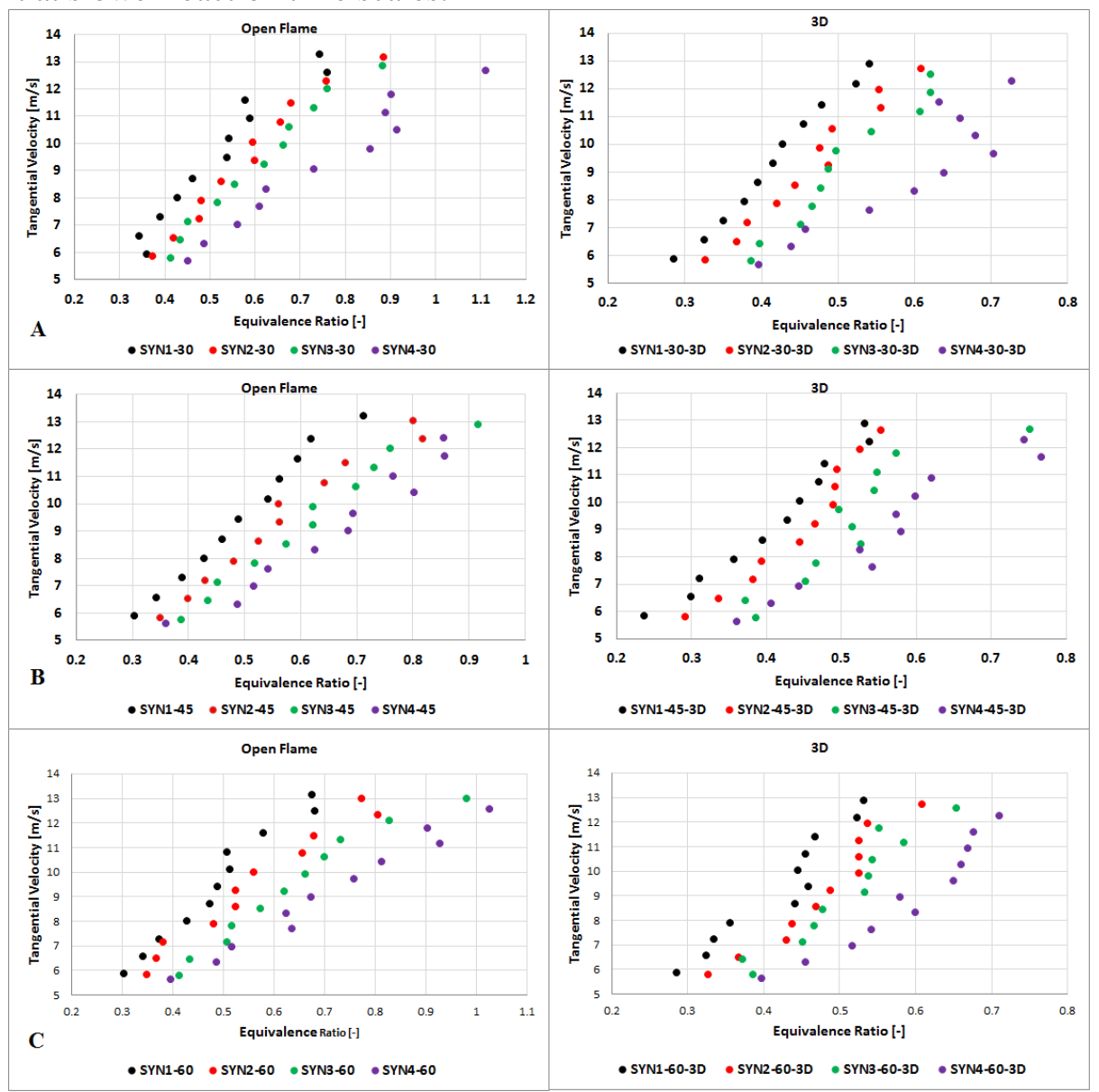

Figure 4: Comparison of blowoff limits, with different nozzle geometries $a, b$ and $c$.

Transition to blowoff was observed to start with the reduction of the flame size to a point of almost disappearance. Once reaching this point, the flame would start oscillating in the transverse direction, with the re-ignition of the blend at low frequencies as a consequence of the recirculation of gases. The 
stronger CRZ observed under isothermal conditions [4] pulls back some of the hot products that will find a point of high interaction with the reactants between the CRZ and the outgoing flow. It has been demonstrated that this region is where the CRZ and the Precessing Vortex Core co-exist [4, 9]. Since their interaction will depend on the strength and shape of the CRZ, therefore, it was expected a greater dependency of the blowoff on the geometry. However, at low flow rates just a slight dependency was observed, Figure 6. Hydrogen content variation, thus the resulting change in $\mathrm{Da}$, was more important to the phenomenon.

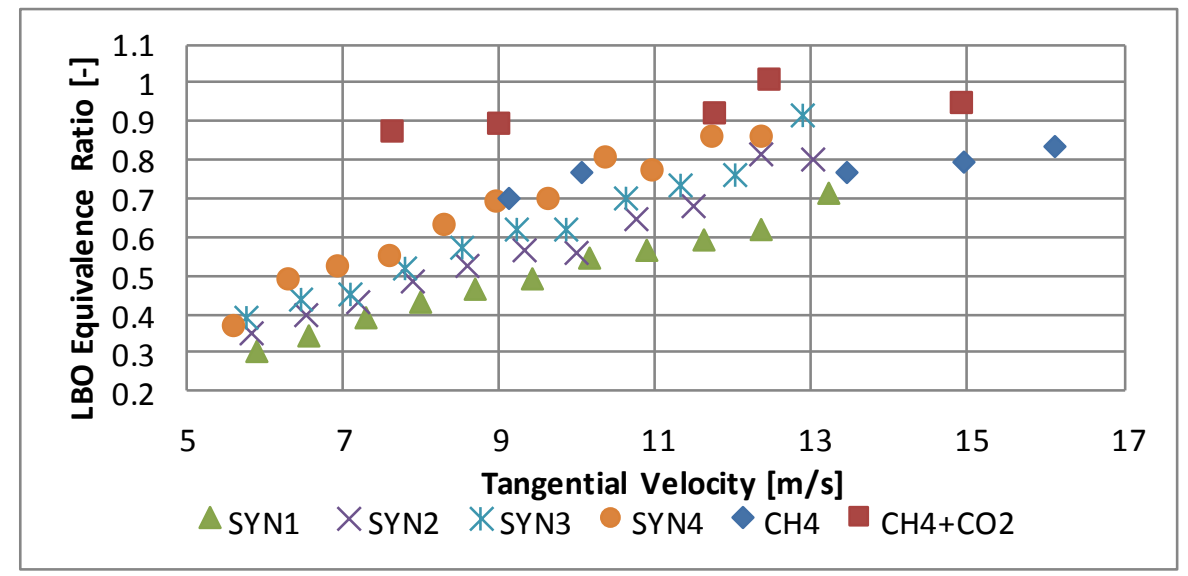

Figure 5: LBO equivalence ratios of different fuel mixtures at same swirl numbers and nozzle $45^{\circ}$.

The results show that the hydrodynamic interaction between the CRZ-PVC plays a minor role in the blowoff for hydrogen enriched blends at these conditions. The influence of the nozzle shows a slight effect for all cases at low and medium flowrates. However with syngas 4, as Re was increased, there was a considerable shift in LBO equivalence ratios using all nozzles as a consequence of the reduction of $\mathrm{H}_{2}$ and distortion of the CRZ, thus CRZ-PVC interaction.
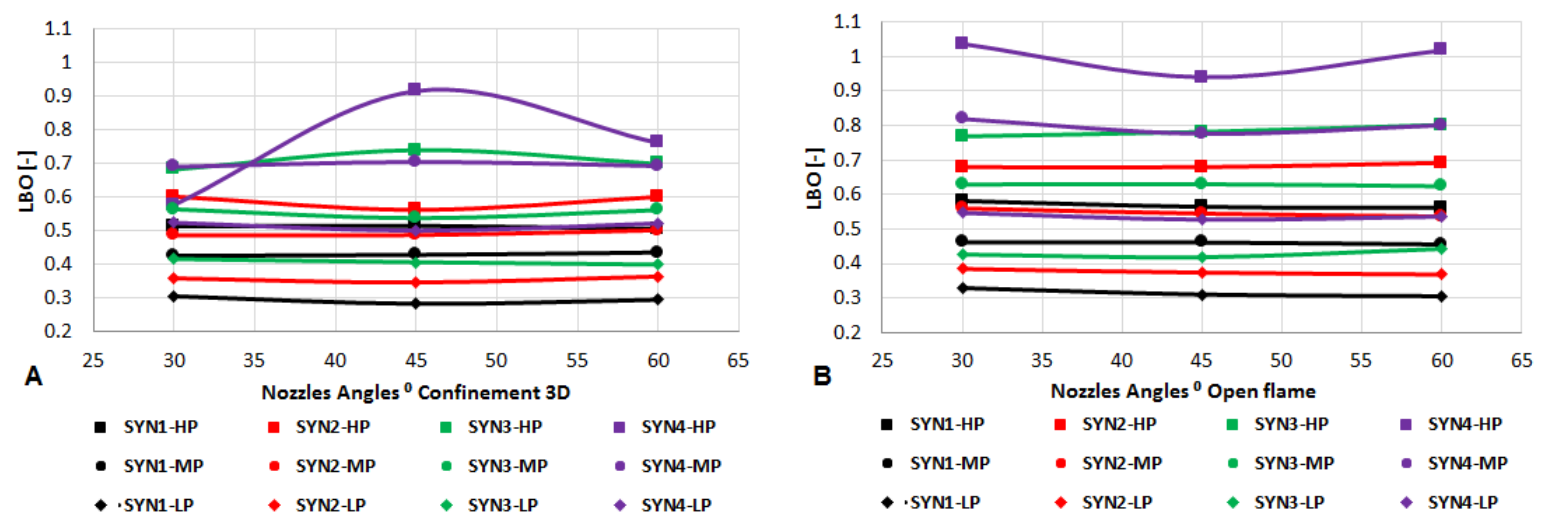

Figure 6 :Comparsion of the effect of outlet nozzle angle on LBO equivalence ratio for all syngases at $L P, M P$ and $L P[K W]$

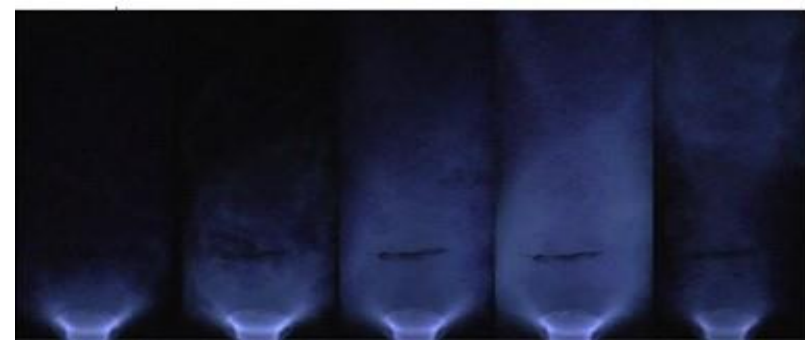

Figure 7: Pulsating flame. Progression of LBO seconds before final onset. $S=1.05, \Phi=0.525$.

Frequency $10 \mathrm{~Hz}$. 
Final blowoff was produced after a longer flame appeared with an intermediate constriction at the centre, Figure 7. The flame showed a cycle of ignition, elongation and quenching just before increasing the flowrate for the final annihilation of the flame. The observed constriction seems to be formed by the reaction of the reactants around the CRZ and a secondary recirculation zone that forms further downstream from the burner mouth. It is believed that this second recirculation, previously observed in other works $[4,22]$. This secondary structure can be defined by the baroclinic depression in the central region caused by the swirling motion, the strength of the main CRZ and acoustics of a $3 / 4$ wave. More research is required on this point.

\section{Numerical results}

Different CRZ boundary contours, Figure 8, turbulence intensity, Da number, stretch factor and turbulent flame speed, Figure 9 , for all syngases using the $45^{\circ}$ nozzle at $7.49 \mathrm{~kW}$ were obtained. The point at which the flow becomes negative diverged from $\mathrm{r} / \mathrm{D}=-0.5$ to 0.5 indicating that the $\mathrm{CRZ}$ is increasing its width whilst the shearing flow is getting slimmer and stronger as a consequence of the greater negativity of the recirculation zone.

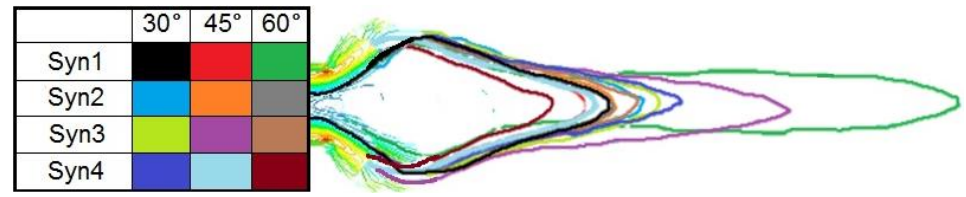

Figure 8: CRZ contours using different syngases.
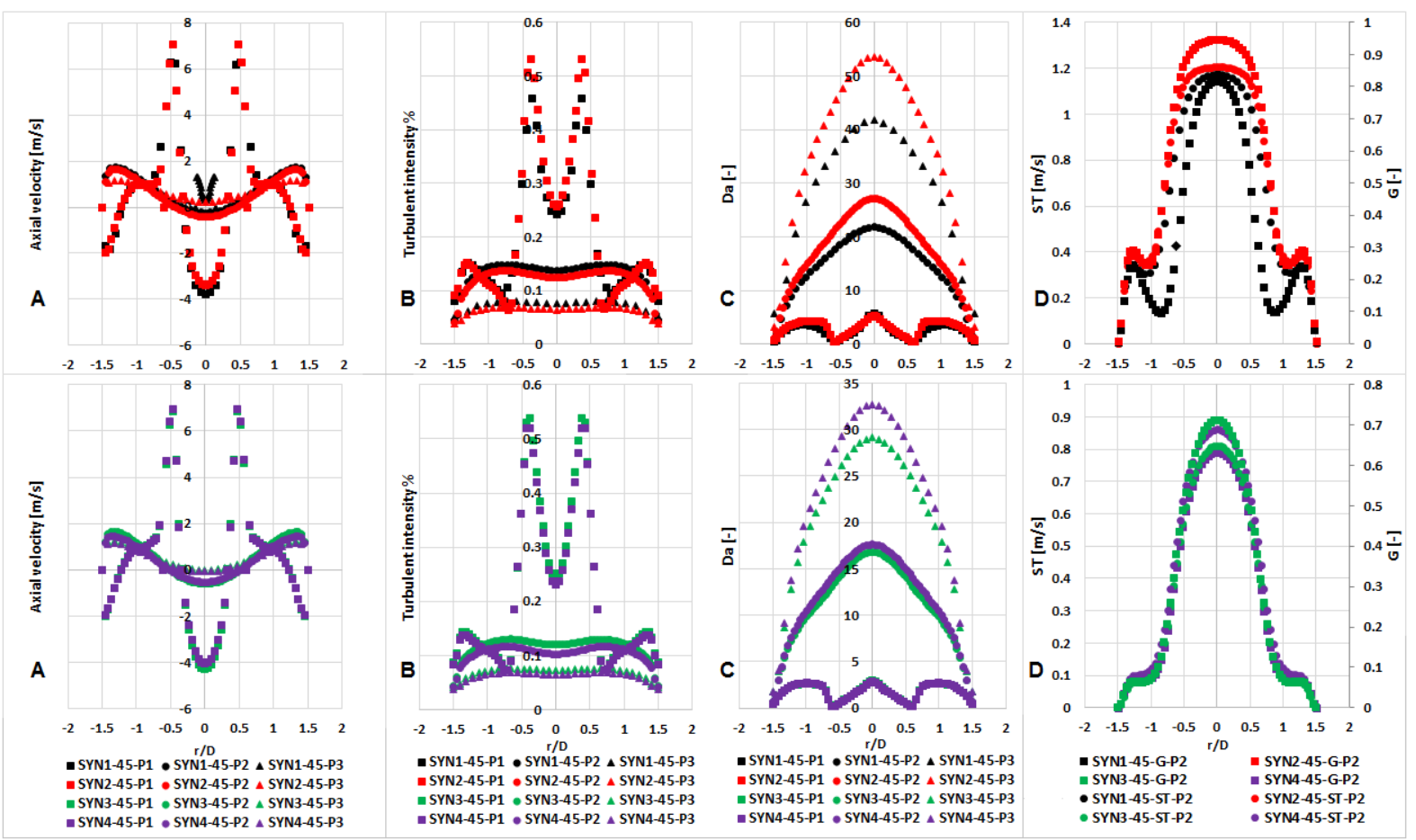

Figure 9: Comparison of (a) axial velocity; (b) turbulent intensity; (c) Damköhler number;

Stretch factor and turbulent flame speed across the flame for all syngases using nozzle $45^{\circ}$ at 3 different planes $P 1, P 2$ and $P 3$ at $X / D=0.00,3.57$ and 7.14 , respectively.

The increase of hydrogen shows an increase in turbulent flame speed and stretch, Figure 9. The Damköhler number increases along the plenum, Figure 9d, due to the decrease in the turbulent intensity and increase of length scales of the flame, Figure 9b. It is in the boundaries of the CRZ where the length scales go to a minimum, thus increasing turbulent intensity and decreasing Da, Figures $9 \mathrm{~b}$ and $9 \mathrm{c}$. Regarding the CRZ the use of different geometrical configurations and fuels have demonstrated that the shape and strength of the structure can change drastically depending on these factors, Figure 8 and Table 3. These findings correlate to previous studies [22]. Comparing the CRZ size, CFD calculations indicate that the use of the $60^{\circ}$ nozzle produces the largest structures, as expected [4]. 
Figure 10 illustrates the axial velocity and turbulent intensity using different nozzles with the same fuel at the same power conditions. The strongest CRZ appears when using the $60^{\circ}$ nozzle, with the highest turbulence intensity. This proves that these geometrical changes can highly impact on the CRZ, thus the PVC and its interaction with the stability of the flow, as observed in the experimental trials.

Table 3. Comparison of the CRZ size of four of syngas using three different angular nozzles

\begin{tabular}{|c|c|c|c|c|c|c|}
\hline Gas & \multicolumn{2}{|c|}{$30^{\circ}$} & \multicolumn{2}{|c|}{$45^{\circ}$} & \multicolumn{2}{|c|}{$60^{\circ}$} \\
\hline No & Width & Length & Width & Length & Width & Length \\
\hline Syn1 & $1.85 D$ & $4.7 D$ & $1.9 D$ & $4.3 D$ & $1.4 \mathrm{D}$ & $9.6 D$ \\
\hline Syn2 & $1.8 D$ & $5.2 D$ & $1.85 D$ & $4.6 D$ & $1.8 D$ & $4.7 D$ \\
\hline Syn3 & $1.9 D$ & $5.4 D$ & $1.9 D$ & $7.2 D$ & $1.8 D$ & $5.2 D$ \\
\hline Syn4 & $1.9 D$ & $5.8 D$ & $1.9 D$ & $4.6 D$ & $1.7 D$ & $4 D$ \\
\hline
\end{tabular}

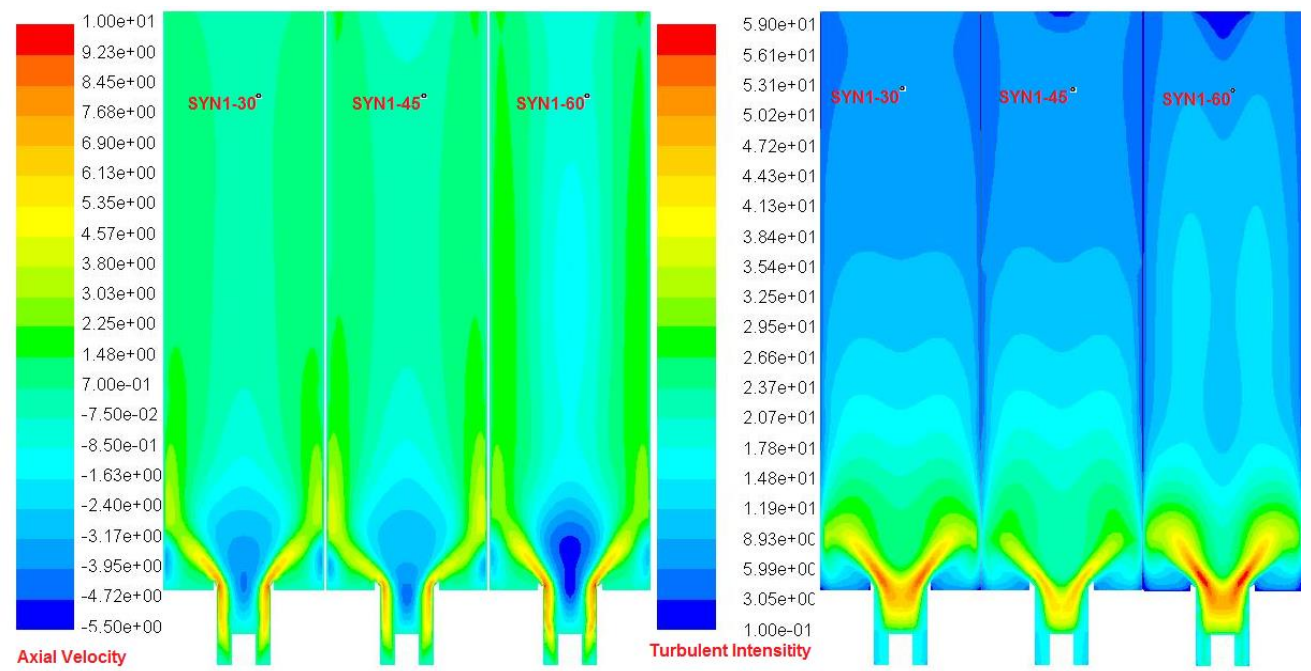

Figure 10: Axial velocity and turbulent intensity using the three nozzles at $7.48 \mathrm{KW}$.

\section{Conclusion}

Experimental tests and numerical simulations have been conducted in an atmospheric, premixed swirl burner to investigate the LBO limit of various syngas mixtures at a moderate swirl number at the same power output using three types of outlet nozzles.

Increasing the mole fraction of $\mathrm{H} 2$ from $25 \%$ to $45 \%$ extended the $\mathrm{LBO}$ limit of a given fuel mixture. This has been previously observed in other experiments and works. There is a small effect of the nozzle angle on the LBO at low flow rates using all mixtures. However, there is a pronounced effect at higher flowrates with low hydrogen content blends. This is assumed to be a consequence of the effect of the CRZ-PVC effect on the flame as a result of a slower chemical reaction. As the $\mathrm{H} 2$ is increased the fast reaction of the molecule reduces any perceptible dependency on the hydrodynamics close to the nozzle. Also, the size of the CRZ is highly affected by the blend. Moreover, these shapes also are affected by the nozzle when using the same compositions, thus denoting how the structure is dependent on the geometry. The length of the CRZ is also a function of the strength of secondary vortices which will impact on the blowoff, strengthening the previous comment.

\section{Acknowledgement}

The authors gratefully acknowledge the support of the Welsh Government Low Carbon Research Institute Programme, the EPSRC (grant no EP/G060053). Mr. Hesham Baej gratefully acknowledges the support of the Libyan Embassy and the Libyan Cultural and Education Bureau in London. 


\section{References}

[1]Karalus, M. 2013. An Investigation of Lean Blowout of Gaseous Fuel Alternatives to Natural Gas. University of Washington.

[2]Syred, N. 2006. A review of oscillation mechanisms and the role of the precessing vortex core (PVC) in swirl combustion systems. Progress in Energy and Combustion Science 32(2), pp. 93-161.

[3] Lefebvre, A.. 1999. Gas Turbine Combustion. Second Edi. USA.

[4] Valera-medina, Agustin, Syred N, B.P. 2013. Central Recirculation Zone analysis using a confined swirl Burner for Terrestrial Energy. J AIAA 29(1), pp. 195-204.

[5] Lieuwen, T. 2012. Unsteady Combustor Physics. USA: Cambridge Press.pp.430.

[6] Longwell, J.P. et al. 1953. Flame Stability in Bluff Body Recirculation Zones. Industrial \& Engineering Chemistry 45(8), pp. 1629-1633.

[7]Shanbhogue, S.J. et al. 2009. Lean blowoff of bluff body stabilized flames: Scaling and dynamics. Progress in Energy and Combustion Science 35(1), pp. 98-120.

[8] Driscoll, J.F. 2008. Turbulent premixed combustion: Flamelet structure and its effect on turbulent burning velocities. Progress in Energy and Combustion Science 34(1), pp. 91-134.

[9]Valera-Medina, a. et al. 2009. Visualisation of isothermal large coherent structures in a swirl burner. Combustion and Flame 156(9), pp. 1723-1734.

[10] N. Peters 2000. Turbulent combustion. Cambridge University Press.

[11]Lieuwen, T. et al. 2008. Fuel Flexibility Influences on Premixed Combustor Blowout, Flashback, Autoignition, and Stability. Journal of Engineering for Gas Turbines and Power 130(1), p. 011506.

[12] Lee, M.C. et al. 2012. Experimental study on the effect of N2, CO2, and steam dilution on the combustion performance of $\mathrm{H} 2$ and $\mathrm{CO}$ synthetic gas in an industrial gas turbine. Fuel 102, pp. 431438.

[13] Strakey, P. et al. 2007. Investigation of the effects of hydrogen addition on lean extinction in a swirl stabilized combustor. Proceedings of the Combustion Institute 31 II, pp. 3173-3180.

[14] Schefer, R.W. et al. 2002. Combustion of hydrogen-enriched methane in a lean premixed swirlstabilized burner. Proceedings of the Combustion Institute 29(1), pp. 843-851.

[15]Baej, H. et al. 2014. Impacts on Blowoff by a Variety of CRZs Using Various Gases for Gas Turbines. Energy Procedia 61, pp. 1606-1609.

[16]Zimont, V. et al. 1998. An Efficient Computational Model for Premixed Turbulent Combustion at High Reynolds Numbers Based on a Turbulent Flame Speed Closure. Journal of Engineering for Gas Turbines and Power 120(3), p. 526.

[17] Date A W 2005. Introduction to Computational Fluid Dynamics. Cambridge University Press.

[18]Poinsot T, V.D. 2005. Theoretical and Numerical Combustion. Edwards ed. USA.

[19] Versteeg HK and Malalasekera W, 1995, An Introduction to Computational Fluid Dynamics The Finite Volume Method, Longman Group Ltd.

[20] Zimont, V.L. 2000. Gas premixed combustion at high turbulence. Turbulent flame closure combustion model. Experimental Thermal and Fluid Science 21(1-3), pp. 179-186.

[21] Vigueras-Zuñiga, M.O. et al. 2012. Studies of the precessing vortex core in swirling flows. Journal of Applied Research and Technology 10(5), pp. 755-765.

[22] Ranga Dinesh, K.K.J. et al. 2013. Burning syngas in a high swirl burner: Effects of fuel composition. International Journal of Hydrogen Energy 38(21), pp. 9028-9042. 